\title{
SIRS o no SIRS: ¿es esa la infección? Una revisión crítica de los criterios de definición de sepsis
}

\author{
Juan C. Jaramillo-Bustamante ${ }^{1,2,3 *}$, Byron E. Piñeres-Olave 4 y Sebastián González-Dambrauskas 3,5 \\ ${ }^{1}$ Unidad de Cuidados Intensivos Pediátricos, Hospital General de Medellín, Medellín, Colombia; ${ }^{2}$ Universidad de Antioquia, Medellín, Colombia; \\ ${ }^{3}$ Red Colaborativa Pediátrica de Latinoamérica (LARed Network), Montevideo, Uruguay; ${ }^{4}$ Unidad de Cuidados Intensivos Pediátricos, Hospital \\ Pablo Tobón Uribe, Medellín, Colombia; ${ }^{5}$ Cuidados Intensivos Pediátricos Especializados, Casa de Galicia, Montevideo, Uruguay
}

\begin{abstract}
Resumen
El enfoque moderno de la sepsis se ha centrado en la creación de consensos globales que utilizan distintos criterios para pesquisarla en forma precoz, con el fin de disminuir la morbimortalidad asociada a ella. Hasta la aparición del tercer y último consenso de adultos (Sepsis-3), el síndrome de respuesta inflamatoria sistémica (SIRS) fue el pilar diagnóstico utilizado por defecto en todas las edades. En Sepsis-3 se decidió retirar el SIRS, lo que generó un debate internacional sobre la oportunidad de dicho cambio. Esta revisión narrativa desarrolla la historia de las distintas definiciones de sepsis centradas en SIRS, las fortalezas, las debilidades y la pertinencia de los distintos elementos que ocasionaron el debate. Dada la ausencia de actualizaciones pediátricas en Sepsis-3, se hace especial énfasis en las implicaciones para las futuras definiciones de sepsis en esta etapa de la vida.
\end{abstract}

Palabras clave: Sepsis. SIRS. qSOFA. Choque séptico. Definición. Pediatría.

\section{SIRS or not SIRS: Is that the infection? A critical review of the sepsis definition criteria}

\begin{abstract}
The modern approach to sepsis has focused on creating a global consensus with different criteria to early investigate it in order to reduce the morbidity and mortality associated with this complex entity. Until the third and last consensus of adults (Sepsis-3), the systemic inflammatory response syndrome (SIRS) was the diagnostic pillar used by default for all ages. In Sepsis-3, it was decided to withdraw the SIRS, which generated an international debate about the timing of such change. This narrative review develops the history of the different definitions of sepsis focused on SIRS, their strengths and weaknesses, and the relevance of the different elements that caused the debate. Given the absence of pediatric updates in Sepsis-3, a particular emphasis is placed on the implications for future definitions of sepsis at this stage of life
\end{abstract}

Key words: Sepsis. SIRS. qSOFA. Septic shock. Definition. Pediatrics.

\section{Correspondencia:}

*Juan C. Jaramillo-Bustamante

E-mail: juancamilojara@gmail.com (http://creativecommons.org/licenses/by-nc-nd/4.0/).
Disponible en internet: 13-11-2020 Bol Med Hosp Infant Mex. 2020;77(6):293-302

www.bmhim.com 
El aumento del conocimiento depende por completo de la existencia del desacuerdo. Karl Raimund Popper (1902-1994)

\section{Introducción}

La palabra griega sepsis (ønభıৎ) ha sobrevivido con más 0 menos el mismo significado por más de 2700 años desde su primera descripción en tiempos de Homero: descomposición o putrefacción ${ }^{1}$. Desde mucho antes de que se supiera algo de microorganismos (era pre-Pasteur) y de la respuesta inmunitaria, el término ya existía y sus manifestaciones clínicas eran conocidas.

Actualmente se sabe que aquella "carne podrida", ligada al accionar de los microorganismos, es el resultado de una compleja interacción de los patógenos y el huésped. A pesar de su longevidad, esta compleja y devastadora enfermedad ha sido elusiva para la medicina debido a las distintas etapas que se han transitado (y aún se transitan) para entenderla y caracterizarla. Por ejemplo, en la evolución histórica de su entendimiento, luego de la aparición de los antibióticos, los pacientes seguían muriendo sépticos (incluso sin patógenos circulando), por lo que los investigadores fueron comprendiendo que también el huésped desempeñaba un papel fundamental en la etiopatogenia ${ }^{2}$. Este síndrome complejo llamado sepsis es el resultado de la desregulación inflamatoria sistémica en respuesta a una infección, que puede poner en riesgo la vida por fallos orgánicos progresivos ${ }^{3}$.

Luego de la aparición de las últimas definiciones de sepsis ${ }^{4}$, en 2016, se generó un intenso debate acerca de la forma en que los profesionales de la salud la definen. Numerosos artículos han puesto a consideración las fortalezas y las debilidades de las distintas definiciones históricas.

El objetivo de esta revisión fue comparar y contrastar la literatura publicada sobre la sepsis y sus definiciones, así como analizar de forma crítica las controversias surgidas, evaluando los posibles alcances de estas en la práctica clínica al atender niños con sepsis.

\section{Llamado de la Organización Mundial de la Salud}

«En mayo de 2017, la Asamblea Mundial de Salud y la Alianza Global de la Sepsis hicieron de esta enfermedad una prioridad sanitaria global (una amenaza mundial para adultos y niños), al adoptar una resolución que urgía a los países miembros de la Organización Mundial de la Salud (OMS) a tomar medidas en la próxima década para mejorar su prevención, su diagnóstico y su tratamiento, reconociendo así que la sepsis es una prioridad en el mundo" ${ }^{5-7}$.

El impacto real de la sepsis a escala mundial se desconoce. En 2016, en una revisión sistemática en población adulta se encontró que, en los últimos 10 años, la tasa de incidencia fue de 437 por 100,000 personas/año para la sepsis (intervalo de confianza del 95\% [IC 95\%]: $334-571 ; t=0.38$ ) y de 270 por 100,000 personas/año para los casos de sepsis grave (IC 95\%: 176-412; $t=$ 0.60 ) en países de altos ingresos económicos, con una mortalidad hospitalaria del $17 \%$ para la sepsis y del $26 \%$ para la sepsis grave durante este período. No se realizaron estimaciones de la incidencia de sepsis en los países con ingresos más bajos (donde viven casi 9 de cada 10 seres humanos), lo que limita la predicción de casos y muertes globales. Sin embargo, a partir de esos datos, una extrapolación tentativa estima 31.5 millones de casos de sepsis y 19.4 millones de sepsis grave, con un potencial de 5.3 millones de muertes anuales ${ }^{8}$.

En la infancia, el escenario epidemiológico es aún más complejo, pues las causas de muerte están codificadas por tradición en un formato que "esconde» la sepsis como causa de muerte. En varios reportes internacionales se evidencia que las infecciones graves continúan siendo la causa principal de morbimortalidad en la edad pediátrica: de las 5.9 millones de muertes que ocurren anualmente en el mundo en niños menores de 5 años, se estima que cerca del $42 \%$ se relacionan con enfermedades infecciosas (neumonía, diarrea, meningitis, septicemia neonatal, malaria, tétanos, tosferina y sarampión) ${ }^{9}$. En 2017, la colaboración Global Burden of Disease Child and Adolescent Health ${ }^{10}$ reportó entre las principales causas de muerte y discapacidad residual en menores de 15 años las infecciones respiratorias bajas, las complicaciones por prematuridad, la asfixia perinatal, la diarrea, la malaria, la desnutrición, la sepsis neonatal, la meningitis y el VIH/sida. Los países donde ocurre la enorme mayoría de eventos son aquellos con menos recursos. En un metaanálisis ${ }^{11}$ se reportaron tasas estimadas anuales de incidencia de 48 por 100,000 (IC 95\%: 27-86) para sepsis y de 22 por 100,000 (IC 95\%: 14-33) para sepsis grave, con un aproximado de 1.2 millones de casos de sepsis y con intervalos de mortalidad del $1-5 \%$ para sepsis y del $9-20 \%$ para sepsis grave. La mayoría de estos decesos se producen por insuficiencia respiratoria, choque o deshidratación, y el espectro de la sepsis es un síndrome clínico probable (y vía final común) en varias de estas etiologías.

Se ha demostrado una enorme disparidad global de la sepsis en cuanto a desenlaces clínicos relevantes. Tras analizar más de 94 estudios y 7561 pacientes, 
Tan, et al. ${ }^{12}$ reportaron que la tasa de mortalidad asociada con sepsis en los países de altos ingresos es del $19 \%$, mientras que en los de bajos ingresos se eleva al $32 \%$. Esto evidencia que la sepsis es una enfermedad socioeconómica.

En 2015 se publicó el primer estudio epidemiológico internacional en sepsis pediátrica grave (SPROUT, Sepsis Prevalence, Outcomes, and Therapies Study $)^{13}$, que involucró 128 unidades de cuidados intensivos (UCl) pediátricas de 26 países (para un total de 6925 ingresos). Se encontró una prevalencia puntual en las UCI pediátricas del 8.2\% (IC 95\%: 7.6-8.9\%) para sepsis grave, con el $25 \%$ de mortalidad hospitalaria y el $24 \%$ en las UCI pediátricas. En los análisis por región, la prevalencia puntual en América del Sur fue más alta, del 16.3\% (IC 95\%: 12.1-21.3\%), con una mortalidad en las UCI pediátricas del $11 \%$. Sin embargo, la población que llega a las UCI pediátricas muestra sesgos, y muchos niños sépticos ni siquiera reciben esos cuidados ${ }^{6}$.

Para cualquier clínico entrenado en la identificación, el diagnóstico y el tratamiento de la sepsis es fácil comprender que el común denominador de todas esas causas infecciosas que determinan la muerte es este mismo síndrome. Sin embargo, la codificación epidemiológica convencional la subestima, pues suelen considerar la sepsis como causa de muerte solo en la etapa neonatal, por lo que se trata como "código basura" en las estadísticas de impacto globales de enfermedades, en las que la mayoría de las muertes se clasifican como "debidas a la infección subyacente» ${ }^{5}$. Por eso, vale la pena preguntarse lo siguiente: ¿de qué muere un gran porcentaje de niños con desnutrición?, ¿de qué mueren los niños con malaria?, ¿de qué mueren los niños con neumonías, meningitis o diarreas, o los que sufren complicaciones debidas al sarampión?, ¿y un niño con trauma grave que sobrevive al siniestro durante su estadía en la $\mathrm{UCl}$ o un enfermo oncológico inmunodeprimido en tratamiento? La respuesta más probable es que un gran porcentaje de ellos (y la verdadera característica unificadora de esas muertes) fallecen por sepsis, el mayor (y menos reconocido) asesino de pacientes pediátricos del mundo ${ }^{14}$. En esta vía de análisis se publicó el más grande estudio epidemiológico de la sepsis en el mundo ${ }^{15}$, que reveló que el panorama puede ser peor de lo que se creía: para 2017 se estimaron 48.9 millones de casos de sepsis (IC 95\%: 38.9-62.9) y 11 millones de muertes (IC 95\%: 10.1-12.0) relacionadas con esta enfermedad, lo que representó el 19.7\% (IC 95\%: 18.2-21.4) de todas las muertes anuales globales.

\section{Problema de las definiciones operativas}

La historia de los consensos de sepsis ha sido, es y será motivo de constante debate.

En 2005 se publicaron las primeras definiciones de sepsis pediátrica ${ }^{16}$ como resultado de un proceso iniciado 3 años antes, durante la International Pediatric Sepsis Consensus Conference, en la que 20 expertos en adultos y niños de Canadá, los Estados Unidos, Francia, Holanda y el Reino Unido consensuaron las definiciones operativas adaptadas para la sepsis. Se incluyó el síndrome de respuesta inflamatoria sistémica (SIRS) y se vinculó con una infección (probable o confirmada) como definitorios de afecciones relacionadas, aunque diferenciadas por los siguientes criterios: sepsis, sepsis grave, choque séptico y disfunción multiorgánica. Llama la atención que dichas definiciones siguen sugiriéndose en publicaciones de referencia de la práctica clínica ${ }^{17,18}$, aunque no fueron diseñadas para el uso clínico. En realidad, los propios autores aclararon que era un trabajo «en progreso" y que esas definiciones surgían para la estandarización de estudios clínicos observacionales (de preferencia grandes y multicéntricos) y de intervenciones terapéuticas dirigidos a mejorar los resultados en la sepsis pediátrica ${ }^{16}$. Goldstein, et al. ${ }^{16}$ tomaron los criterios de SIRS de los estudios originales de sepsis en adultos ${ }^{19,20}$ y los adaptaron, considerando que los puntos de corte para las frecuencias cardíaca y respiratoria que definían SIRS en la edad pediátrica debían reformularse. Propusieron, entonces, los puntos de corte publicados en el artículo original. Sin embargo, ¿de dónde surgieron estos puntos de corte?, ¿qué evidencia sustentaba estos valores para la definición de sepsis en 2002-2005?

Los puntos de corte utilizados para definir taquicardia y polipnea quizás no eran correctos, por lo que se definiría como «anormalidad» algo que no lo es. Investigaciones posteriores a 2005 mostraron que las tablas de los percentiles de los valores fisiológicos que diferencian un SIRS positivo de uno negativo, y que se utilizan en las guías habituales de práctica clínica, pueden no condecirse con los valores normales para distintas edades pediátricas. Fleming, et al. ${ }^{21}$ sistematizaron la evidencia publicada hasta 2011 en cuanto a los valores de las frecuencias cardíaca y respiratoria en niños sanos de hasta 18 años, y encontraron que los valores tomados como normales en las gráficas necesitaban actualizarse. Incluso, para definir la hipotensión, otros investigadores encontraron que los valores normales para algunas poblaciones son mayores que los rangos sugeridos por algunas guías de 
resucitación, como por ejemplo la guía PALS (Pediatric Advanced Life Support) ${ }^{22}$. La distribución normal puede diferir según el sitio donde los parámetros vitales sean medidos, como se demuestra en distintos análisis multicéntricos de cientos de miles de niños $\operatorname{sanos}^{23}$ atendidos en salas de hospitalización ${ }^{24}$ o en el contexto de una UCl pediátrica ${ }^{25}$. También puede variar por grupos de enfermedades, como mostraron Eytan, et al., en $2017^{26}$. Incluso, la dependencia entre la frecuencia cardíaca y la temperatura corporal se encuentra sujeta a modificaciones distintas en niños hospitalizados en sala y en aquellos atendidos en atención primaria o emergencias ${ }^{27}$. Lo anterior sugiere que utilizar los puntos de corte actualmente recomendados por las guías que incluyen el SIRS puede inducir a un error.

Desde 1992, sepsis y SIRS quedaron ligados a los primeros dos consensos de poblaciones adultas (conocidos hoy como Sepsis 1 y 2) ${ }^{19,28}$. Los médicos adoptaron, para el uso clínico, algunas definiciones que son, cuando menos, discutibles, y que fueron diseñadas para la investigación, no para la atención de pacientes. A su vez, estas definiciones alimentaron las principales guías de manejo de sepsis (léase las campañas de Sobreviviendo a la Sepsis). De esta forma, los clínicos empezaron a asumir que el diagnóstico de sepsis y choque séptico se basa en un conjunto de criterios (SIRS) comunes también a otras enfermedades, como pancreatitis, grandes quemaduras y posoperatorios cardiovasculares, entre otras; que podrían estar presentes o no en el paciente con sepsis; que no eran específicos y que podrían inducir a un falso diagnóstico. Las críticas al respecto fueron crecientes.

En 2013, algunos líderes de opinión del cuidado intensivo de adultos ${ }^{29}$ plantearon profundos interrogantes a la utilidad del SIRS como pilar en la definición de sepsis: su alta sensibilidad, ya que más del $90 \%$ de los pacientes que ingresan a $\mathrm{UCl}$ pueden presentarlo, sin existir una etiología infecciosa; el hecho de que algunas personas pueden «responder» a una infección con las variables que se tienen en cuenta en la definición; la incapacidad de conocer el peso que la infección aportaba al SIRS y que la "antiinflamación» también es un espectro posible en la sepsis. Posteriormente, un extenso estudio retrospectivo epidemiológico realizado en Australia y Nueva Zelanda ${ }^{30}$ en 172 UCI puso en entredicho la sensibilidad del SIRS al encontrar que dejaba fuera uno de cada ocho pacientes con infección y disfunción orgánica. Como solución a lo anterior, se planteó la necesidad de definir la sepsis como una respuesta sistémica a la infección con la presencia de algún grado de disfunción orgánica. Para tratar de detectar aquellos pacientes con mayor mortalidad, se cambió el enfoque usando puntajes específicos de fallo orgánico. Esta fue la base de Sepsis-3, la tercera definición de la sepsis.

\section{Final del SIRS: Sepsis-3 y el debate internacional}

En 2016 se publicó el Tercer Consenso Internacional para Sepsis y Choque Séptico (Sepsis-3) ${ }^{4}$, en el cual el SIRS desapareció de las definiciones y quedó desvinculado de la sepsis. Este grupo de consenso contó con reuniones en 2014-2015 de 19 expertos de sociedades de cuidado crítico norteamericanas y europeas, y partió de las premisas de que, a la fecha, la sepsis (entendida como un síndrome) no contaba con una prueba diagnóstica validada, que el SIRS mostraba una baja validez discriminante (falsos positivos) y una baja validez convergente (falsos negativos), y que se requería una «herramienta más operativa, más pragmática, que diferenciara la infección no complicada de una verdadera sepsis», entendida esta última como «una respuesta del hospedero a la infección, no regulada y aberrante». Para hacer operativo lo anterior se escogió la escala SOFA (Sequential Organ Failure Assessment) ${ }^{31}$ y se creó una versión resumida de tamización (quick SOFA o qSOFA) que evalúa la respiración, el estado mental y la presión arterial sistólica. Así las cosas, desaparece el concepto de sepsis grave, la sepsis se considera como una infección sospechada o comprobada con disfunción orgánica (dada por un aumento en el puntaje SOFA), y el choque séptico como una sepsis con anormalidades circulatorias y metabólicas. Cabe notar que dicho consenso fue avalado por 31 sociedades científicas (listadas al final del articulo original), pero otras (especialmente de atención primaria y de urgencias, como el American College of Chest Physicians, el American College of Emergency Physicians, la Society for Academic Emergency Medicine y el Latin American Sepsis Institute) no lo han hecho ${ }^{32-36}$. Las principales razones para ello fueron no haber incluido a médicos de urgencias ni a países de bajos y medianos ingresos en su elaboración. Además, estas definiciones no se habían validado prospectivamente en una población amplia ni se habían estudiado en el servicio de urgencias, en enfermos prehospitalarios ni en pacientes hospitalizados fuera de UCI. Lo anterior, debido a que se pensó que podría ir en detrimento de la necesidad de los países de bajos y medianos ingresos de aumentar la conciencia sobre la enfermedad, y 
por razones operativas con el uso de la hiperlactatemia como criterio.

Luego de su publicación, Sepsis-3 inquietó a un sector de la comunidad académica respecto a los posibles efectos deletéreos de retirar el SIRS de la definición de la enfermedad ${ }^{37}$ y ello motivó una avalancha de editoriales a favor ${ }^{38-42}$ y en contra ${ }^{43-48}$ de su base conceptual, dividiendo al mundo académico, a la fecha, en dos orillas aparentemente irreconciliables: Sepsis-3 vs. SIRS. A tal punto se ha llegado que muchas discusiones han revelado una profunda carga emocional (baste con citar algunos títulos de dichos editoriales para entenderlo: «la medicina imprecisa» ${ }^{38}$, «de amor y felicidad» 39 , «para SIRS con amor: una carta abierta» 46 , «viendo toda la imagen» ${ }^{41}$, «lo bueno, lo malo y lo feo» ${ }^{44}$, «infielmente tuyo $»^{42}$ ).

En la Tabla 1 se hace una comparación de los elementos básicos de cada aproximación, y a continuación se resumen los argumentos esgrimidos por los detractores de Sepsis-3:

- EI SIRS ha demostrado su utilidad clínica y académica por más de 20 años.

- La escala SOFA no está ampliamente aceptada ni se utiliza de manera generalizada en las $\mathrm{UCl}$, y menos aún en los servicios de urgencias. Además, su uso en la sepsis no ha sido validado e incluye elementos de laboratorio no tan «disponibles» en todos los escenarios clínicos ni en todos los perfiles epidemiológicos.

- Entendiendo esta enfermedad como un espectro que va desde la infección hasta la muerte, la disfunción orgánica estaría «más allá» que el SIRS en dicha gama, lo que podría generar un retraso en la identificación y en la instauración de las medidas terapéuticas necesarias en pacientes con enfermedad grave que aún no hayan desarrollado la disfunción de un órgano.

- Cuando se hace referencia al choque, Sepsis-3 solo incluiría aquel que cursa con un aumento del lactato e hipotensión (conocido en la literatura como choque con disoxia tisular), pero no el choque criptogénico (entendido como la presencia de hiperlactatemia sin hipotensión) ni el choque vasopléjico (que hace referencia a la presencia de hipotensión sin hiperlactatemia) ${ }^{47}$.

- A varios autores les parece que se estuviera proponiendo un cambio de definición de un síndrome clínico por otro, sin que se origine nada nuevo desde el punto de vista conceptual.

- Mencionar que la intención de abandonar una herramienta clínica de tan amplio recorrido como ha sido el SIRS (que usa criterios «familiares, universales,
Tabla 1. Elementos básicos de SIRS y de Sepsis-3

\begin{tabular}{|l|l}
\hline SIRS & Sepsis-3 \\
\hline - Amplio recorrido y & - Mejor especificidad \\
conocido por los clínicos & - Consenso amplio basado en \\
- Sensibilidad aceptable & extensas bases de datos de \\
- Mejor para reconocer la & UCI \\
enfermedad en estadios & - Predice mejor la mortalidad en \\
iniciales & escenarios UCI \\
- Se encuentra uantes» & - Puede tener menor sensibilidad \\
que la disfunción & fuera de UCI \\
orgánica en el espectro & - La escala SOFA no es \\
de la sepsis & comúnmente utilizada \\
- Pobre especificidad & - Puede no incluir algunos tipos \\
- Sin validación clínica & de choque que cursan sin \\
extensa & aumento del lactato \\
& - La hipotensión es tardía en los \\
& niños \\
\hline & - No incluyó opinión de médicos \\
de urgencias ni de países de
\end{tabular}

SIRS: síndrome de respuesta inflamatoria sistémica; UCI: unidad de cuidados intensivos; SOFA: Sequential Organ Failure Assessment.

oportunos y sensibles») ${ }^{46}$ requiere contundencia en los datos que lo motiven y prudencia en la toma de decisiones, pues un cambio tan drástico y abrupto puede acarrear profundas consecuencias en la atención y en la investigación.

- La construcción de Sepsis-3 derivó de un análisis retrospectivo de bases de datos en $\mathrm{UCl}$, sin que mediara una validación posterior. Por tanto, predice la mortalidad y la estancia en la $\mathrm{UCl}$, pero no ha probado reducir la mortalidad. Es decir, pareciera que aumenta la especificidad a expensas de la sensibilidad, que para muchos es precisamente la virtud que ha tenido el SIRS: centrarse en la sensibilidad. Es así como un reciente metaanálisis ${ }^{49}$ mostró una mejor sensibilidad del SIRS frente a la escala qSOFA para el diagnóstico de sepsis, mientras que la qSOFA fue ligeramente superior para predecir la mortalidad.

\section{Sepsis-3 se extiende a la pediatría}

Sepsis-3 excluyó a neonatos y niños mayores de su definición, lo que dejó una brecha en el grupo etario con mayor incidencia de sepsis. Como era lógico de esperar, un cambio mayor en la medicina de adultos tiende a influir en la pediatría.

Aunque el concepto de «respuesta desregulada del huésped» es atractivo y posee un fundamento biológico, no está bien definido ni es apto para la medición cuantitativa o cualitativa. El fuerte enfoque de Sepsis-3 
en puntajes de disfunción orgánica plantea problemas para la aplicación en entornos donde los recursos de laboratorio no están disponibles. Además, los criterios son difíciles de aplicar en los servicios de urgencias (primer lugar para la identificación del paciente séptico), donde la toma de decisiones a menudo se basa en una evaluación clínica rápida, incluso antes de que las pruebas de laboratorio estén disponibles.

Varios editoriales ${ }^{6,50-54}$ llamaron la atención sobre la importancia de tener definiciones precisas en pediatría y sugirieron un mayor protagonismo de la disfunción orgánica (como en Sepsis-3). Además, se abrió el debate a la necesidad de revisar las actuales definiciones de sepsis, con un deseo manifiesto de que estuvieran unificadas con las de la medicina de adultos.

Tanto en la práctica clínica como en investigaciones recientes se han evidenciado también las debilidades del SIRS para pediatría. Luego de la publicación de los resultados globales obtenidos en el estudio SPROUT ${ }^{13}$ se realizaron algunos análisis de subgrupos pertinentes a esta discusión. El primero se centró en la discordancia existente entre las definiciones clínicas y las de investigación (o del consenso): de los 6925 pacientes analizados, 706 se diagnosticaron como sepsis grave (bien fuera por cumplir los criterios del consenso de 2015 o como un diagnóstico dado por el médico tratante), por lo que solo se encontró acuerdo en el $42.6 \%$ entre el concepto clínico y el del consenso ${ }^{55}$. Así, el $19.4 \%$ fue diagnosticado solo por la clínica, sin cumplir criterios del consenso, mientras que el $38 \%$ los cumplían sin que el concepto clínico fuera concordante con ello. Visto de otro modo, de los 438 pacientes con diagnóstico clínico de sepsis grave, el $31 \%$ no cumplía las definiciones del consenso. Por otro lado, de los 569 con sepsis grave por el consenso, el $47 \%$ no contaba con esa «percepción» clínica de su médico tratante. Esto revela conflictos no solo en la actuación terapéutica ante estos pacientes, sino también en su posible inclusión en protocolos de investigación.

El segundo análisis derivado del SPROUT se centró en el concepto de disfunción orgánica nueva o progresiva, que podría entenderse como un fenotipo especial de la enfermedad ${ }^{56}$. De los 567 pacientes con sepsis grave, 384 presentaron disfunción orgánica asociada en los 7 días posteriores a su ingreso; de ellos, 57 no la presentaron al ingreso, pero la desarrollaron en el seguimiento (disfunción nueva), y de los 327 con alguna disfunción en el día 1, en 91 se documentó «progresión» (disfunción progresiva). Esto significa que se documentó el fenotipo de disfunción orgánica nueva o progresiva en 148 casos (26\%). Al realizar los análisis respectivos se encontró una mayor necesidad de soporte respiratorio mecánico, infusión de vasoactivos, uso de hemoderivados, insulina y terapias de reemplazo renal en este grupo; además, presentó mayor mortalidad, estancia hospitalaria y en la $\mathrm{UCl}$ pediátrica, y mayor discapacidad residual al alta (con significación estadística). La mortalidad se elevaba de forma gradual a medida que aumentaba el número de órganos en disfunción, lo que sugiere que la disfunción de órganos es relevante en el espectro clínico de la sepsis.

Se cuenta con estudios que evaluaron si la disfunción orgánica podría ser también el centro de la definición en sepsis pediátrica (Tabla 2) ${ }^{57-63}$.

Es de notar que los estudios mencionados son análisis retrospectivos de bases de datos (la mayoría en $\mathrm{UCl}$ pediátricas) de pacientes cuyo primer desenlace ya ocurrió (necesidad de UCI pediátrica o mortalidad), con resultados dispares en las diferentes cohortes analizadas. Esto complica la extrapolación de los hallazgos a todos los escenarios en los cuales se debe diagnosticar a tiempo la enfermedad ${ }^{64}$. Además, el contraste existente entre la mortalidad por esta causa en países de bajos y moderados ingresos, así como la baja producción en investigación que allí se da, hacen también que los resultados y las recomendaciones derivadas tal vez estén alejados de la verdadera realidad ${ }^{11,64,65}$.

El reto de lograr el diagnóstico temprano para un tratamiento oportuno y efectivo sigue vigente ${ }^{66}$, y aunque cada vez se cuenta con más herramientas paraclínicas con miras a lograr una identificación precoz de la disfunción orgánica y de la posible etiología de la enfermedad, no hay duda de que el primer eslabón de la cadena debería ser, principalmente, clínico y basado en condiciones objetivas más que en «recomendaciones de expertos». De ahí la relevancia de la actual discusión y la necesidad de un conocimiento global por parte de la comunidad médica.

\section{Direcciones futuras}

Sin duda, todo cambio de paradigma ocasiona resistencia y debate; sin embargo, esto último es precisamente el motor que promueve el avance y el refinamiento en la ciencia. Por ello, es inevitable plantearse algunas interrogantes que han ido surgiendo respecto al diagnóstico de sepsis en pediatría:

- ¿Será el momento de tomar distancia de las definiciones usadas en la medicina de adultos, de no tratar de adaptarlas, ajustarlas o forzarlas a la realidad 
Tabla 2. Estudios que usaron la disfunción orgánica como predictor de muerte en la sepsis pediátrica

\begin{tabular}{|c|c|c|c|c|c|}
\hline Estudio & Análisis & $\mathbf{n}$ & Objetivo & Resultados & Conclusiones \\
\hline $\begin{array}{l}\text { Leclerc, et al. }{ }^{57} \\
\text { Nueve UCI } \\
\text { pediátricas } \\
\text { francófonas }\end{array}$ & $\begin{array}{l}\text { Retrospectivo, } \\
\text { de registros } \\
2006-2007\end{array}$ & 862 & $\begin{array}{l}\text { Capacidad } \\
\text { predictiva de } \\
\text { mortalidad de } \\
\text { PELOD-2 }\end{array}$ & $\begin{array}{l}\text { AUC d1PELOD-2 de } 0.91 \text { (IC 95\%: } 0.86-0.96 \text { ) } \\
\text { en sospecha de infección, de } 0.88 \text { (IC } 95 \% \text { : } \\
0.79-0.96 \text { ) en presencia de hipotensión } \\
\text { sistólica con hiperlactatemia, de } 0.91 \text { (IC } \\
95 \%: 0.85-0.97 \text { ) en presencia de } \\
\text { hipotensión media con hiperlactatemia y } \\
\text { de } 0.82 \text { (IC } 95 \% \text { : } 0.76-0.87 \text { ) para qPELOD-2 } \\
\text { d1PELOD-2 } \geq 8 \text { se correspondía con un } \\
\text { riesgo de mortalidad } \geq 9.3 \% \text { en niños con } \\
\text { sospecha de infección; VPP } 35.4 \% \text { y VPN } \\
98.8 \% \\
\text { qPELOD-2 } 22 \text { se correlacionaba con una } \\
\text { mortalidad del } 19.8 \% \text { (en presencia de } \\
\text { hipotensión sistólica; VPP } 25.3 \% \text { y VPN } \\
97.2 \% \text { ) o del } 15.9 \% \text { (en presencia de } \\
\text { hipotensión media; VPP } 21.1 \% \text { y VPN 97.7\%) }\end{array}$ & $\begin{array}{l}\text { Escalas altamente } \\
\text { predictoras de } \\
\text { mortalidad }\end{array}$ \\
\hline $\begin{array}{l}\text { Matics y } \\
\text { Sanchez-Pinto }{ }^{58} \\
\text { Una UCl } \\
\text { pediátrica de los } \\
\text { Estados Unidos }\end{array}$ & $\begin{array}{l}\text { Retrospectivo, } \\
\text { de registros } \\
2009-2016\end{array}$ & 4217 & $\begin{array}{l}\text { Validación } \\
\text { pSOFA }\end{array}$ & $\begin{array}{l}\text { El diagnóstico de sepsis aumentaba la } \\
\text { probabilidad de muerte (OR: } 18 ; \text { IC } 95 \% \text { : } \\
11-28 \text { ). De las } 229 \text { muertes, el } 65 \% \\
\text { cumplían el criterio de sepsis o choque } \\
\text { séptico }\end{array}$ & $\begin{array}{l}\text { pSOFA es una escala } \\
\text { prometedora en el } \\
\text { diagnóstico de sepsis }\end{array}$ \\
\hline $\begin{array}{l}\text { Schlapbach, } \\
\text { et al. }{ }^{59} \\
\text { Base de datos } \\
\text { ANZICS }\end{array}$ & $\begin{array}{l}\text { Retrospectivo, } \\
\text { de registros } \\
2000-2016\end{array}$ & 2594 & $\begin{array}{l}\text { Comparación } \\
\text { entre SIRS, } \\
\text { SOFA y } \\
\text { PELOD }\end{array}$ & $\begin{array}{l}\text { SOFA y PELOD tuvieron un mejor } \\
\text { comportamiento que SIRS, con un AUC > } \\
0.8 \text {. } \\
\text { qSOFA fue similar a SIRS }\end{array}$ & $\begin{array}{l}\text { SIRS carece de } \\
\text { especificidad para } \\
\text { identificar niños con } \\
\text { infección con un riesgo } \\
\text { sustancialmente mayor } \\
\text { de mortalidad }\end{array}$ \\
\hline $\begin{array}{l}\text { Zallocco et al. }{ }^{60} \\
\text { Hospital italiano }\end{array}$ & $\begin{array}{l}\text { Retrospectivo, } \\
\text { de registros } \\
2006-2016\end{array}$ & 89 & $\begin{array}{l}\text { Capacidad } \\
\text { discriminativa } \\
\text { de qSOFA }\end{array}$ & $\begin{array}{l}\text { S: } 46 \% \text { (IC } 95 \%: 27-67 \% \text { ) } \\
\text { E: } 74 \% \text { (IC } 95 \%: 62-85 \%) \\
\text { VPP: } 43 \% \text { (IC } 95 \%: 34-52 \% \text { ), } \\
\text { VPN: } 77 \% \text { (IC } 95 \%: 71-82 \% \text { ) } \\
\text { AUC para un valor } \geq 2: 0.602 \text { (IC } 95 \% \text { : } \\
0.492-0.705 \text { ) }\end{array}$ & $\begin{array}{l}\text { qSOFA mostró una baja } \\
\text { precisión para identificar } \\
\text { a los niños en la sala de } \\
\text { pediatría con riesgo de } \\
\text { sepsis grave }\end{array}$ \\
\hline $\begin{array}{l}\text { van Nassau, } \\
\text { et al. } .^{61} \\
\text { Sala de } \\
\text { urgencias en los } \\
\text { Países Bajos }\end{array}$ & $\begin{array}{l}\text { Retrospectivo, } \\
\text { de registros } \\
\text { 2013-2018 }\end{array}$ & 864 & $\begin{array}{l}\text { Capacidad } \\
\text { predictiva } \\
\text { para ingreso a } \\
\text { UCI pediátrica } \\
\text { o muerte en } \\
\text { pacientes con } \\
\text { sospecha de } \\
\text { infección } \\
\text { bacteriana }\end{array}$ & $\begin{array}{l}\text { qSOFA mostró ser mejor predictor para } \\
\text { ingreso a UCI pediátrica o muerte (AUC: } \\
0.72 \text {; IC 95\%: } 0.57-0.86 \text { ) que SIRS (AUC: } \\
0.64 \text {; IC } 95 \% \text { : } 0.53-0.74 \text { ) y qPELOD-2 (AUC: } \\
0.60 \text {; IC 95\%: } 0.45-0.76 \text { ) }\end{array}$ & $\begin{array}{l}\text { qSOFA fue mejor } \\
\text { predictor que SIRS y } \\
\text { qPELOD-2 para ingreso a } \\
\text { UCI pediátrica o muerte. } \\
\text { La adición de la } \\
\text { medición de lactato a } \\
\text { qSOFA no mejoró su } \\
\text { desempeño }\end{array}$ \\
\hline $\begin{array}{l}\text { Görges, et al. }{ }^{62} \\
\text { Base datos } \\
\text { canadiense }\end{array}$ & $\begin{array}{l}\text { Retrospectivo, } \\
\text { de registros } \\
2009-2014\end{array}$ & 42,196 & $\begin{array}{l}\text { Validación } \\
\text { externa de } \\
\text { qPELOD2 }\end{array}$ & $\begin{array}{l}\text { qPELOD- } 2 \geq 2 \text { : mortalidad del } 13.4 \% \\
\text { S: } 50.5 \% \\
\text { E: } 85.5 \% \\
\text { VPP } 13.4 \% \\
\text { VPN: } 97.5 \% \\
\text { Falsos negativos: } 49.5 \%\end{array}$ & $\begin{array}{l}\text { Determinar qué variables } \\
\text { son importantes al } \\
\text { elaborar una puntuación } \\
\text { de sepsis de utilidad } \\
\text { universal requiere más } \\
\text { trabajo, y ponen en duda } \\
\text { la hipotensión como } \\
\text { herramienta para } \\
\text { identificar niños con } \\
\text { sepsis en mayor riesgo }\end{array}$ \\
\hline $\begin{array}{l}\text { Sankar, et al. }{ }^{63} \\
\text { UCI pediátrica } \\
\text { en India }\end{array}$ & $\begin{array}{l}\text { Retrospectivo, } \\
\text { de registros } \\
\text { 2014-2017 }\end{array}$ & 216 & $\begin{array}{l}\text { Capacidad } \\
\text { discriminativa } \\
\text { SIRS vs. } \\
\text { Sepsis-3 }\end{array}$ & $\begin{array}{l}\text { Mortalidad global: } 42.5 \% \\
\text { Mortalidad SIRS } 37.5 \% \text { vs. mortalidad } \\
\text { Sepsis-3 } 48.5 \% \text { (RR: } 1.3 \text {; IC } 95 \% \text { : } \\
0.94-1.75 ; p=0.1 \text { ) } \\
\text { pSOFA a las } 24 \text { h fue mayor en Sepsis-3 } \\
\text { que en SIRS ( } 9 \text { vs. } 6 ; p=0.0008 \text { ), así } \\
\text { como PELOD fue mayor en Sepsis-3 que } \\
\text { en SIRS ( } 12 \text { vs. } 11 ; p=0.04) \text {. } \\
\text { Para diagnóstico de choque, SOFA: S } \\
80 \% \text { y E } 73 \% \\
\text { Para diagnóstico de choque, PELOD: S } \\
80 \% \text { y E } 75 \%\end{array}$ & $\begin{array}{l}\text { Sepsis-3 solo identificó } \\
\text { niños más graves en } \\
\text { choque séptico (solo el } \\
48 \% \text { de los clasificados } \\
\text { así por SIRS), lo que } \\
\text { podría inducir un } \\
\text { subdiagnóstico y limitar } \\
\text { su uso en pediatría }\end{array}$ \\
\hline
\end{tabular}

ANZICS: Australian and New Zealand Intensive Care Society; AUC: área bajo la curva; d1PELOD-2: el primer día de PELOD-2; E: especificidad; IC 95\%: intervalo de confianza del 95\%; OR: odds ratio; PELOD-2: Pediatric Logistic Organ Dysfunction-2; pSOFA: adaptación pediátrica de SOFA; qPELOD-2: quickPELOD-2; RR: riesgo relativo; S: sensibilidad; SIRS: systemic inflammatory response syndrome; SOFA: Sequential Organ Failure Assessment; UCI: unidad de cuidados intensivos; VPN: valor predictivo negativo; VPP: valor predictivo positivo. 
pediátrica, y mejor construir unas nacidas desde las observaciones y necesidades propias? Algo así como lo que ocurrió con la definición del síndrome de dificultad respiratoria aguda, cuando se tomó distancia con las definiciones de Berlín y se diseñó un concepto propio para la pediatría ${ }^{67}$. Más allá, ¿serán los casos pediátricos con sepsis que se atienden comparables a los casos de las guías internacionales, sabiendo que las investigaciones al respecto se realizan mayoritariamente en países de altos ingre$\operatorname{sos}^{11,68}$, mientras que la mayor carga de la enfermedad se encuentra en países de medianos y bajos ingresos?

- En lugar de abandonar el concepto de SIRS, pero considerando sus debilidades, ¿por qué no mejor refinarlo?, pues pareciera que los clínicos «con experiencia» saben en quiénes usar y en quiénes no usar los criterios de SIRS al clasificar como sepsis a un paciente.

- La introducción de algunos criterios basados en disfunción orgánica y en pruebas de laboratorio poco accesibles en algunos escenarios clínicos, ¿mejorará de forma efectiva el desempeño diagnóstico en sepsis?, ¿o será de utilidad contar con unos criterios muy sensibles, para lograr un diagnóstico precoz en salas de urgencias y hospitalización, y otros muy específicos, para un diagnóstico preciso que permita afinar el pronóstico y aplicarlo en escenarios de $\mathrm{UCl}$ pediátricas?

Claramente, se deberían implementar múltiples herramientas de diferente complejidad para la identificación de sepsis, que puedan ser complementarias y utilizarse con sentido común, sin caer en «recetas de cocina». En un extremo de dicha complejidad se deben citar los avances importantes que se han logrado mediante el uso de algoritmos de inteligencia artificial, alimentados con información procedente de historias clínicas electrónicas para predecir el desarrollo de sepsis en algunas poblaciones ${ }^{69-71}$. Esta tecnología avanza a pasos agigantados en el terreno clínico, y seguramente en un futuro no muy lejano será aplicada a pie de cama del paciente, ya que se nutre de variables fisiológicas y analiza y predice la trayectoria clínica según el análisis de millones de datos. Lo anterior ha comenzado a facilitar el reconocimiento de los distintos fenotipos de sepsis, algunos de los cuales podrían no ser detectados por los métodos de cribado habitua$\mathrm{les}^{72}$. La inteligencia artificial fue noticia en 2018, cuando algunos algoritmos mostraron ser superiores al proceso de decisiones de manejo lideradas por un médico, por lo que este recurso diagnóstico va a tomar relevancia conforme avance la investigación ${ }^{73}$.

Otro punto de gran interés, y olvidado en los consensos, es la relevancia de la percepción clínica de los médicos: la "corazonada» (gut feeling) del profesional que se encuentra con un paciente cursando una infección y "no lo ve bien", siente que "algo anda mal», aunque no siempre pueda ser confirmado por variables fisiológicas alteradas. Existe evidencia de que este juicio clínico, junto con la percepción de los padres acerca del estado de su hijo, son buenos predictores de presencia de sepsis ${ }^{74,75}$. A diferencia de las poblaciones adultas, en pediatría los padres son los principales cuidadores del niño, los que más lo conocen y también los aliados del médico en el reconocimiento de la sepsis. Varios estudios enfatizan que escuchar las preocupaciones de los padres puede ser de gran ayuda en el reconocimiento de la sepsis y no debe soslayarse su importancia. Aunque se necesitan futuras investigaciones para determinar el preciso valor diagnóstico en diferentes escenarios sanitarios, y a pesar de algunos reportes no tan alentadores sobre su utilidad ${ }^{76}$, las armas clínicas deben refinarse y la percepción de las familias debería incluirse en la evaluación y la tamización diagnóstica ${ }^{77}$.

Se requiere que todos estos interrogantes y direcciones futuras empiecen a clarificarse con los trabajos de grupos crecientes de investigación, consorcios y redes de investigación pediátricas ${ }^{78}$. Estos grupos, que trabajan en los diferentes contextos donde los niños se enferman de sepsis, con particular énfasis en aquellas regiones del mundo que más lo necesitan, y centran sus objetivos en la consecución de datos clínicos del día a día de los pacientes, ayudarán a construir sus propias realidades, siguiendo la evolución natural de la enfermedad y no partiendo del fatídico desenlace. Para un problema global se necesita cooperación global y trabajo a gran escala que permita validar las definiciones futuras. Muchos de estos grupos, que trabajan en diversos escenarios, ya han incluido la sepsis dentro de sus prioridades de investigación, por lo que, seguramente, el fruto de nuevas investigaciones en la materia se podrá notar en breve ${ }^{79-81}$.

Esta interesante controversia frente a lo que es la sepsis pediátrica no ha finalizado. En los próximos años seremos testigos y artífices de los refinamientos teóricos tendientes a mejorar nuestras prácticas frente a esta enfermedad. Entretanto, en el intermezzo de esta discusión, los médicos que atendemos día a día a niños con infecciones graves seguiremos a pie de cama, afinando el juicio clínico junto a las familias y 
utilizando, en conjunto, las mejores herramientas disponibles para tratarlos.

\section{Responsabilidades éticas}

Protección de personas y animales. Los autores declaran que para esta investigación no se han realizado experimentos en seres humanos ni en animales.

Confidencialidad de los datos. Los autores declaran que han seguido los protocolos de su centro de trabajo sobre la publicación de datos de pacientes.

Derecho a la privacidad y consentimiento informado. Los autores han obtenido el consentimiento informado de los pacientes y/o sujetos referidos en el artículo. Este documento obra en poder del autor de correspondencia.

\section{Financiamiento}

Ninguno.

\section{Conflicto de intereses}

Los autores declaran no tener ningún conflicto de intereses.

\section{Bibliografía}

1. Geroulanos S, Douka ET. Historical perspective of the word "sepsis". Intensive Care Med. 2006;32:2077.

2. Angus DC, van der Poll T. Severe sepsis and septic shock. N Engl J Med. 2013;369:840-51.

3. Bone RC. The sepsis syndrome. Definition and general approach to management. Clin Chest Med. 1996;17:175-81.

4. Singer M, Deutschman CS, Seymour CW, Shankar-Hari M, Annane D, Bauer M, et al. The third international consensus definitions for sepsis and septic shock (Sepsis-3). JAMA. 2016;315:801-10.

5. Reinhart K, Daniels R, Kissoon N, Machado FR, Schachter RD, Finfer $\mathrm{S}$. Recognizing sepsis as a global health priority - a WHO resolution. N Engl J Med. 2017;377:414-7.

6. Schlapbach LJ, Kissoon N. Defining pediatric sepsis. JAMA Pediatr. 2018;172:312-4.

7. Zick M. WHO adopts a resolution on sepsis. Berlin: Global Sepsis Alliance. 2017. Disponible en: https://www.global-sepsis-alliance.org/ news/2017/5/26/wha-adopts-resolution-on-sepsis.

8. Fleischmann C, Scherag A, Adhikari NKJ, Hartog CS, Tsaganos T, Schlattmann $P$, et al. Assessment of global incidence and mortality of hospital-treated sepsis. Current estimates and limitations. Am J Respir Crit Care Med. 2016;193:259-72.

9. Liu L, Oza S, Hogan D, Chu Y, Perin J, Zhu J, et al. Global, regional, and national causes of under-5 mortality in 2000-15: an updated systematic analysis with implications for the sustainable development goals. Lancet. 2016;388:3027-35.

10. Global Burden of Disease Child and Adolescent Health Collaboration; Kassebaum N, Kyu HH, Zoeckler L, Olsen HE, Thomas K, Pinho C, et al. Child and adolescent health from 1990 to 2015: findings from the global burden of diseases, injuries, and risk factors 2015 study. JAMA Pediatr. 2017;171:573-92.

11. Fleischmann-Struzek C, Goldfarb DM, Schlattmann P, Schlapbach LJ, Reinhart K, Kissoon N. The global burden of paediatric and neonatal sepsis: a systematic review. Lancet Respir Med. 2018;6:223-30.

12. Tan B, Wong JJ, Sultana R, Koh JCJW, Jit M, Mok YH, et al. Global case-fatality rates in pediatric severe sepsis and septic shock: a syste- matic review and meta-analysis. JAMA Pediatr. 2019;173:352-62

13. Weiss SL, Fitzgerald JC, Pappachan J, Wheeler D, Jaramillo-Bustamante JC, Salloo A, et al. Global epidemiology of pediatric severe sepsis: the sepsis prevalence, outcomes, and therapies study. Am J Respir Crit Care Med. 2015;191:1147-57.

14. Kissoon N, Uyeki TM. Sepsis and the global burden of disease in children. JAMA Pediatr. 2016;170:107-8.

15. Rudd KE, Johnson SC, Agesa KM, Shackelford KA, Tsoi D, Kievlan DR, et al. Global, regional, and national sepsis incidence and mortality, 1990-2017: analysis for the global burden of disease study. Lancet. 2020;395:200-11.

16. Goldstein B, Giroir B, Randolph A. International pediatric sepsis consensus conference: definitions for sepsis and organ dysfunction in pediatrics. Pediatr Crit Care Med. 2005;6:2-8.

17. Thomas, NJ, Tamburro, RF, Rajasekaran, S, Fitzgerald, JC, Weiss, SL, Hall, MW. Bacterial sepsis. En: Morrison WE, McMillan KLN, Shaffner DH, editores. Rogers' Handbook of pediatric intensive care. Philadelphia: Wolters Kluwer Health; 2017. p. 635.

18. Alder CN, Sandquist M, Wong H.R. Sepsis. En: Fuhrman BP, Zimmerman JJ, editores. Fuhrman and Zimmerman's Pediatric critical care. Philadelphia: Elsevier; 2017. p. 1960.

19. American College of Chest Physicians/Society of Critical Care Medicine Consensus Conference: definitions for sepsis and organ failure and guidelines for the use of innovative therapies in sepsis. Crit Care Med. 1992;20:864-74.

20. Bone RC, Sprung CL, Sibbald WJ. Definitions for sepsis and organ failure. Crit Care Med. 1992;20:724-6.

21. Fleming S, Thompson M, Stevens R, Heneghan C, Plüddemann A Maconochie I, et al. Normal ranges of heart rate and respiratory rate in children from birth to 18 years of age: a systematic review of observational studies. Lancet. 2011;377:1011-8.

22. Chan SS, Cattermole GN, Leung PYM, Mak PSK, Graham CA, Rainer TH Validation of the APLS age-based vital signs reference ranges in a Chinese population. Resuscitation. 2011;82:891-5.

23. Sepanski RJ, Godambe SA, Zaritsky AL. Pediatric vital sign distribution derived from a multi-centered emergency department database. Front Pediatr. 2018;6:1-11.

24. Bonafide CP, Brady PW, Keren R, Conway PH, Marsolo K, Daymont C. Development of heart and respiratory rate percentile curves for hospitalized children. Pediatrics. 2013;131:e1150-7.

25. Eytan D, Goodwin AJ, Greer R, Guerguerian AM, Laussen PC. Heart rate and blood pressure centile curves and distributions by the age of hospitalized critically ill children. Front Pediatr. 2017;5:1-8.

26. Eytan D, Goodwin AJ, Greer R, Guerguerian AM, Mazwi M, Laussen PC. Distributions and behavior of vital signs in critically ill children by admission diagnosis. Pediatr Crit Care Med. 2018;19:115-24.

27. Daymont $\mathrm{C}$, Bonafide CP, Brady PW. Heart rates in hospitalized children by age and body temperature. Pediatrics. 2015;135:e1173-81.

28. Levy MM, Fink MP, Marshall JC, Abraham E, Angus D, Cook D, et al. 2001 SCCM/ESICM/ACCP/ATS/SIS International Sepsis Definitions Conference. Intensive Care Med. 2003;29:530-8.

29. Vincent JL, Opal SM, Marshall JC, Tracey KJ. Sepsis definitions: time for change. Lancet. 2013;381:774-5.

30. Kaukonen KM, Bailey M, Pilcher D, Cooper DJ, Bellomo R. Systemic inflammatory response syndrome criteria in defining severe sepsis. N Engl J Med. 2015;372:1629-38.

31. Vincent JL, de Mendonça A, Cantraine F, Moreno R, Takala J, Suter PM, et al. Use of the SOFA score to assess the incidence of organ dysfunction/failure in intensive care units: results of a multicenter, prospective study. Working group on "sepsis-related problems" of the European Society of Intensive Care Medicine. Crit Care Med. 1998;26:1793-800.

32. Machado FR, Nsutebu E, AbDulaziz S, Daniels R, Finfer S, Kissoon N, et al. Sepsis 3 from the perspective of clinicians and quality improvement initiatives. J Crit Care. 2017;40:315-7.

33. Rezaie S. Rebelem. Sepsis 3.0. 2019. Disponible en: http://rebelem.com/ sepsis-3-0.

34. Al-Salamah T, Winters ME. Saudi Society of Emergency Medicine. Sepsis-3, what's new. Disponible en: http://sasem.org/sepsis-3-whats-new.

35. Faust JS. No SIRS; Quick SOFA instead. Ann Emerg Med. 2016;67:A15-9.

36. Slesinger TL, Dubensky L. American College of Emergency Physicians. Sepsis-3, a new definition. Solutions or new problems? 2016. Disponible en: https://www.acep.org/how-we-serve/sections/quality-improvement--patient-safety/newsletters/july-2016/sepsis--3-a-new-definition.-solutions-or-new-problems.

37. Inoue K, Takano H. Sepsis definitions. Lancet. 2013;381:2249-50.

38. Deutschman CS. Imprecise medicine: the limitations of Sepsis-3. Crit Care Med. 2016;44:857-8.

39. Deutschman CS. Sepsis-3: of love and bliss. Crit Care Med. 2017;45:739-40.

40. Singer $M$. The new sepsis consensus definitions (Sepsis-3): the good the not-so-bad, and the actually-quite-pretty. Intensive Care Med. 2016;42:2027-9. 
41. Deutschman CS. Sepsis-3: seeing the entire picture. Crit Care Med. 2017:45:1567-9.

42. Landis RC, Durandy Y. Dear SIRS...unfaithfully yours. Anaesth Intensive Care. 2018:46:553-640.

43. Farkas J. PulmCrit (EMCrit). Top ten problems with the new sepsis definition. 2016. Disponible en: https://emcrit.org/pulmcrit/problems-sepsis-3-definition.

44. Sprung $\mathrm{CL}$, Schein RMH, Balk RA. The new sepsis consensus definitions: the good, the bad and the ugly. Intensive Care Med. 2016;42:2024-6.

45. Simpson SQ. New sepsis criteria. Chest. 2016;149:1117-8.

46. Sprung CL, Schein RMH, Balk RA. To SIRS with love - an open letter. Crit Care Med. 2017;45:736-8

47. Carneiro AH, Póvoa P, Gomes JA. Dear Sepsis-3, we are sorry to say that we don't like you. Rev Bras Ter Intensiva. 2017;29:4-8.

48. Simpson SQ. SIRS in the time of Sepsis-3. Chest. 2018;153:34-8.

49. Serafim R, Gomes JA, Salluh J, Póvoa P. A comparison of the quick-S FA and systemic inflammatory response syndrome criteria for the diagnosis of sepsis and prediction of mortality. Chest. 2018:153:646-55.

50. Piva JP, Garcia PCR. Sepsis: from the stone age to nowadays without a precise definition. Pediatr Crit Care Med. 2016;17:794-5.

51. Workman JK, Larsen GY. Searching for a pediatric severe sepsis phenotype: are we there yet? Pediatr Crit Care Med. 2017;18:82-3

52. Shime N, Kawasaki T, Nakagawa S. Proposal of a new pediatric sequential organ failure assessment score for possible validation. Pediatr Crit Care Med. 2017;18:98-9.

53. Schlapbach LJ. Time for Sepsis-3 in children? Pediatr Crit Care Med. 2017; $18: 805-6$.

54. Schlapbach LJ, Berger C, Aebi C, Agyeman PKA, Swiss Pediatric Sepsis Study. SIRS in the time of Sepsis-3: what about the children? Chest. 2018; $153: 1512$.

55. Weiss SL, Fitzgerald JC, Maffei FA, Kane JM, Rodriguez-Nunez A Hsing DD, et al. Discordant identification of pediatric severe sepsis by research and clinical definitions in the SPROUT international point prevalence study. Crit Care. 2015;19:1-10.

56. Lin JC, Spinella PC, Fitzgerald JC, Tucci M, Bush JL, Nadkarni VM, et al New or progressive multiple organ dysfunction syndrome in pediatric severe sepsis: a sepsis phenotype with higher morbidity and mortality. Pediatr Crit Care Med. 2017;18:8-16.

57. Leclerc F, Duhamel A, Deken V, Grandbastien B, Leteurtre S, Groupe Francophone de Réanimation et Urgences Pédiatriques. Can the Pediatric Logistic Organ Dysfunction-2 score on day 1 be used in clinical criteria for sepsis in children? Pediatr Crit Care Med. 2017:18:758-63.

58. Matics TJ, Sanchez-Pinto LN. Adaptation and validation of a pediatric sequential organ failure assessment score and evaluation of the Sepsis-3 definitions in critically ill children. JAMA Pediatr. 2017;171:e172352.

59. Schlapbach LJ, Straney L, Bellomo R, MacLaren G, Pilcher D. Prognostic accuracy of age-adapted SOFA, SIRS, PELOD-2, and qSOFA for in-hospital mortality among children with suspected infection admitted to the intensive care unit. Intensive Care Med. 2018;44:179-88.

60. Zallocco F, Osimani P, Carloni I, Romagnoli V, Angeloni S, Cazzato S Assessment of clinical outcome of children with sepsis outside the intensive care unit. Eur J Pediatr. 2018:177:1775-83.

61. Van Nassau SC, van Beek RH, Driessen GJ, Hazelzet JA, van Wering HM, Boeddha NP. Translating Sepsis-3 criteria in children: prognostic accuracy of age-adjusted quick SOFA score in children visiting the emergency department with suspected bacterial infection. Front Pediatr. 2018;6:1-7.

62. Görges M, Peters C, Murthy S, Pi S, Kissoon N. External validation of the "quick" Pediatric Logistic Organ Dysfunction-2 score using a large North American cohort of critically ill children with suspected infection. Pediatr Crit Care Med. 2018;19:1114-9.
63. Sankar J, Dhochak N, Kumar K, Singh M, Sankar MJ, Lodha R Comparison of International Pediatric Sepsis Consensus Conference versus Sepsis-3 definitions for children presenting with septic shock to a tertiary care center in India: a retrospective study. Pediatr Crit Care Med. 2019;20:e122-9.

64. Obonyo NG, Schlapbach LJ, Fraser JF. Sepsis: changing definitions, unchanging treatment. Front Pediatr. 2018:6:1-5.

65. Rudd KE, Kissoon N, Limmathurotsakul D, Bory S, Mutahunga B, Seymour CW, et al. The global burden of sepsis: barriers and potential solutions. Crit Care. 2018;22:1-11.

66. Lynn LA. The diagnosis of sepsis revisited - a challenge for young medical scientists in the $21^{\text {st }}$ century. Patient Saf Surg. 2014;8:1-5.

67. Khemani RG, Smith LS, Zimmerman JJ, Erickson S; Pediatric Acute Lung Injury Consensus Conference Group. Pediatric acute respiratory distress syndrome: definition, incidence, and epidemiology. Proceedings from the Pediatric Acute Lung Injury Consensus Conference. Pediatr Crit Care Med. 2015;16:S23-40.

68. Schlapbach LJ, Argent A. Applying Sepsis-3 criteria for septic shock to children - not as shocking as at first sight? Pediatr Crit Care Med. 2019;20:299-300.

69. Le S, Hoffman J, Barton C, Fitzgerald JC, Allen A, Pellegrini E, et al. Pediatric severe sepsis prediction using machine learning. Front Pediatr. 2019;7:1-8.

70. Guirgis FW, Jones L, Esma R, Weiss A, McCurdy K, Ferreira J, et al. Managing sepsis: electronic recognition, rapid response teams, and standardized care save lives. J Crit Care. 2017;40:296-302.

71. Kamaleswaran R, Akbilgic O, Hallman MA, West AN, Davis RL, Shah SH. Applying artificial intelligence to identify physiomarkers predicting severe sepsis in the PICU. Pediatr Crit Care Med. 2018;19:e495-503.

72. Seymour CW, Kennedy JN, Wang S, Chang C-CH, Elliott CF, Xu Z, et al. Derivation, validation, and potential treatment implications of novel clinical phenotypes for sepsis. JAMA. 2019;321:2003-17.

73. Komorowski M, Celi LA, Badawi O, Gordon AC, Faisal AA. The artificial intelligence clinician learns optimal treatment strategies for sepsis in intensive care. Nat Med. 2018;24:1716-20.

74. Van den Bruel A, Aertgeerts B, Bruyninckx R, Aerts M, Buntinx F. Signs and symptoms for diagnosis of serious infections in children: a prospective study in primary care. Br J Gen Pract. 2007;57:538-46.

75. Van den Bruel A, Thompson M, Buntinx F, Mant D. Clinicians' gut feeling about serious infections in children: observational study. BMJ. 2012;345:e6144.

76. Urbane UN, Gaidule-Logina D, Gardovska D, Pavare J. Value of parental concern and clinician's gut feeling in recognition of serious bacterial infections: a prospective observational study. BMC Pediatr. 2019;19:1-8.

77. Harley A, Latour JM, Schlapbach LJ. The role of parental concerns in the recognition of sepsis in children: a literature review. Front Pediatr. 2019;7:1-10.

78. González-Dambrauskas S, Jaramillo-Bustamante JC, Díaz F. No one is better than all together: the role of networks in pediatric intensive care. Rev Bras Ter Intensiva. 2019;31:277-81

79. Bressan S, Titomanlio L, Gomez B, Mintegi S, Gervaix A, Parri N, et al Research priorities for European paediatric emergency medicine. Arch Dis Child. 2019;104:869-73.

80. Stoner MJ, Mahajan P, Bressan S, Lam SHF, Chumpitazi CE, Kornblith $\mathrm{AE}$, et al. Pediatric emergency care research networks: a research agenda. Acad Emerg Med. 2018;25:1336-44.

81. Von Saint André-von Arnim AO, Attebery J, Kortz TB, Kissoon N, Molyneux EM, Musa NL, et al. Challenges and priorities for pediatric critical care clinician-researchers in low- and middle-income countries. Front Pediatr. 2017;5:1-6. 